\title{
Aspectos epidemiológicos da malária no Amazonas
}

\author{
José João Ferraroni ( \\ Jack Hayes $(t *)$
}

\begin{abstract}
RESUMo
São apresentados os dados de que a infecção malárica no Amazonas vem decrescendo desde 1973, exceto nas localidades de construçâo ou abertura das estradas BR-174 (Manaus/Boa Vista) e BR-319 (Manaus/Porto Velho). Na primeira a malária vem apresentando um foco endêmico, com casos de $\mathbf{P}$. falciparum resistente a drogas e Anopheles darlingi exibindo um comportamento de resistência ao DDT. Desde 1974 a maioria dos casos de infecção vem sendo tratada nos ambulatórios e enfermarias do Hospital de Moléstias Tropicais de Manaus, de onde originam a maioria dos dados, os quais săo apresentados mensal e anualmente dando a espécie de plasmódio, a idade e o sexo dos indivíduos. Verificou-se que mais de $56 \%$ dos casos de malária diagnosticados no referido hospital foram oriundos da BR-174, tornando esta área muito importante para o estudo desta protozoose.
\end{abstract}

\section{INTRODUÇÃ̃o}

A malária tem sido considerada hiperendêmica no Amazonas desde 1972 (Ministério da Saúde, 1973). Desde essa época o número de casos relatados pela SUCAM (Superintendência das Campanhas Médicas, órgão do Ministério da Saúde) tem declinado sensivelmente, de 15.921 casos de infecções em 1972 para 4.543 em 1977. Uma exceção no declínio da incidência do número de casos tem ocorrido nas regiōes de abertura das estradas BR-174 (Manaus/Boa Vista) e BR-319 (Manaus/Porto Velho). Os dados de 1977 representados no relatório anual da SUCAM indicam que mais de $50 \%$ dos casos de malária ocorridos no Estado do Amazonas, neste ano foram oriundos das populações residentes nas proximidades das referidas rodovias.

O Estado do Amazonas tinha uma popula. cão estimada em aproximadamente 1.237.916 habitantes em 1977, sendo que 600.810 vi- viam na área urbana e 637.106 na área rurai (Anuário Estatístico do Amazonas, 1975).

A BR-174 (Manaus/Boa Vista) com uma extensão de aproximadamente $776 \mathrm{~km}$, foi aberta oficialmente para o tráfego em março de 1976. O trecho compreendido entre os km 99 e 200, ou seja do rio Urubu até a re. serva Indigena Waimiri Atroari, foi aberto para a colonização em 1973 (ver mapa). Os colonizadores mudaram-se para aquela área nos últimos 4 anos. Este trecho da estrada recentemente entregue à população, tem sido um foco constante de malária desde o inicio. Resistência de malária à drogas é co mum nessa localidade, onde mais de $80 \%$ das cepas de Plasmodium falciparum são resistentes às cloroquinas (Ferraroni et al., 1977). Amostras das espécies de Anopheles darlingi, o principal vetor da área, tem revelado um comportamento de resistência ao DDT (Hayes \& Charlwood, 1977). Esta combinação de fatores faz com que esta região seja muito importante para estudos, uma vez aue o número de casos de malária tem se mantido e às vezes aumentado, apesar dos €sforços da SUCAM, desde o princípio da colonização da área.

Este trabalho faz referência a natureza dos casos de malária representados pelos prontuários dos pacientes hospitalizados e admissöes, assim como aos casos atendidos nos ambulatórios do Hospital de Moléstias Tropicais. A SUCAM tem pessoal técnico sediado no referido hospital para pesquisa de plasmódio, de rotina, em todos os casos febris que ali dão entrada. Desde abril de 1974, quando foi inaugurado o Hospital de Mo léstias Tropicais de Manaus a maioria do número de casos de malária vêm sendo drenados para ele, assim como praticamente todos

( $\left.{ }^{\circ}\right)$ Instituto Nacional de Pesquisas da Amazônia e Hospital de Moléstias Tropicais de Manaus.

(“) Instituo Nacional de Pesquisas da Amazônia, Manaus. 
os casos em que se justifique a hospitalização. Os casos de malária nos militares (soldados e o pessoal civil trabalhando para os militares) foram obtidos no Hospital Geral cie Manaus, onde são também, internados de rotina, pacientes com infecção malárica.

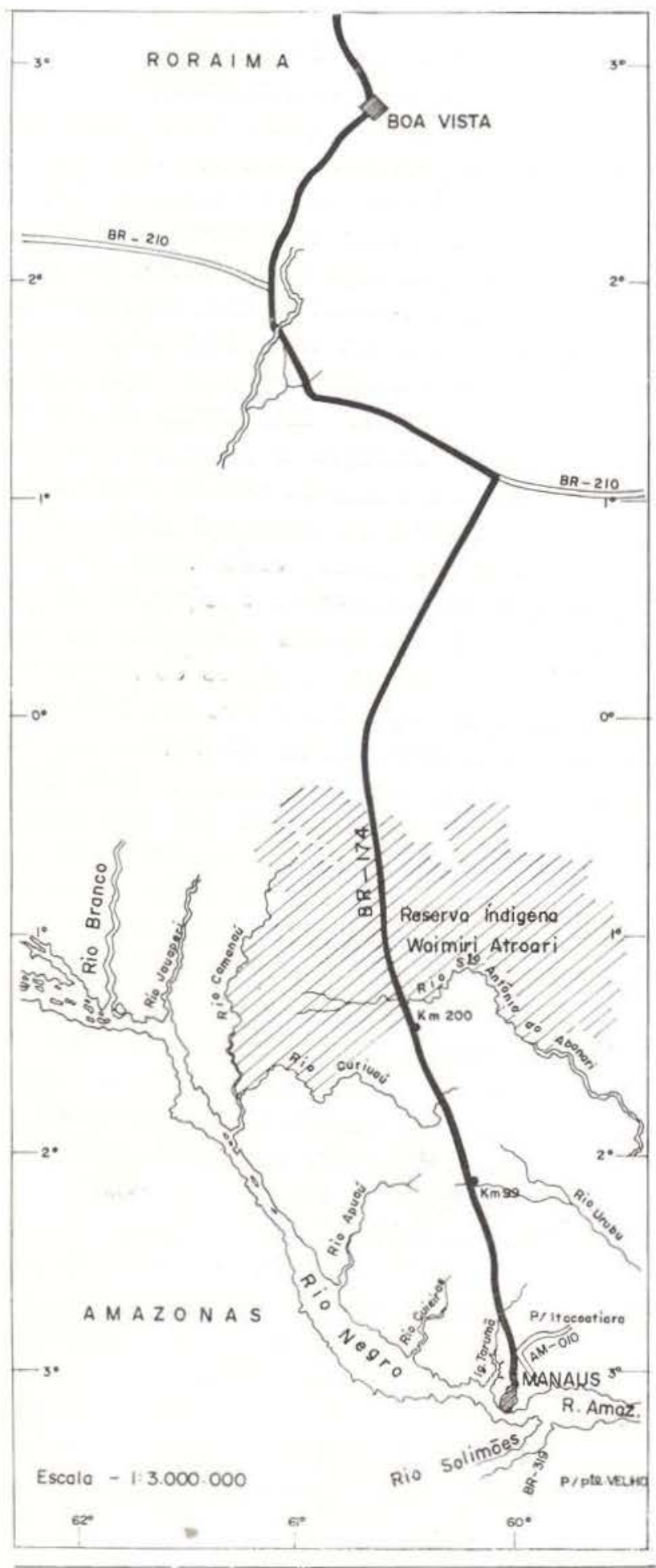

Mapa-Localizaçâo geográfica da BR-174

\section{MATERIAIS E MÉTODOS}

A determinação dos casos de malária foi realizado segundo os padröes recomendados pela Organização Mundial da Saúde (O.M.S., 1975). As amostras de sangue em lâminas, tanto o esfregaço como a gota espessa foram corados pelo método de Giemsa. O esfregaço antes de corado era fixado com metanol. A designação,,$++++++\mathrm{e}++++$ se guem os padrões da O.M.S. e significam: + . é igual a aproximadamente 01 parasita por campo em gota espessa;,++ 02 a 20 parasitas por campo em gota espessa;,+++ 21 a ¿00 parasitas por campo em gota espessa e ++++ mais de 200 parasitas por campo em gota espessa.

Os dados demográficos foram obtidos do Anuário Estatístico do Amazonas e do Censo Demográfico do IBGE. Os dados do hospital foram conseguidos no SAME. Os casos diagnosticados năo hospitalizados foram obtidos no registro da SUCAM no Hospital de Moléstias Tropicais de Manaus. Obtivemos os dados dos militares no Hospital Militar. Os dados metereológicos foram oriundos da Estação Metereológica da Reserva Ducke, que se situa a $30 \mathrm{~km}$ no sentido leste de Manaus $\epsilon$ os dados metereológicos de Manaus, do 1965 a 1975, foram originados da Estação Metereológica da Igreja São José.

\section{Resultados E discussão}

A abertura da BR-174 (Manaus/Boa Vista) para a sua colonização acima do rio Urubu do km 99 ao 200 ocorreu em 1974. Este foi o mesmo ano em que o Hospital de Moléstias Tropicais de Manaus foi aberto para o tratamento de todas as doenças tropicais do Amazonas. As estatísticas da SUCAM mostram um declínio em todos os casos de malária no Amazonas com exceção àqueles registrados nas margens da BR-174, o qual tem se mantiao e até apresentado aumento nos anos mais recentes. Os dados do Hospital de Moléstias Tropicais de Manaus (Tabela 1) indicam que em 1976, 67\% dos casos de malária vivax e $72 \%$ dos casos de falciparum foram oriundos da BR-174 e em 1977 essas proporções foram de $54 \%$ para o $P$. vivax e $73 \%$ para o $P$. falci- 
TABELA 1 - Proporção de casos de Malária da BR-174 (Manaus/Boa Vista) diagnosticados no Hospital de Moléstias Tropicais de Manaus, Amazonas Brasil nos anos de $1976^{\star} \cdot 1977^{\star}$.

\section{6}

\begin{tabular}{|c|c|c|c|c|c|c|c|c|c|c|c|c|}
\hline \multicolumn{7}{|c|}{ Plasmodium vivax } & \multicolumn{6}{|c|}{ Plasmodium falciparum } \\
\hline Origem & Masc & $\%$ & Fem. & $\%$ & Total & $\%$ & Masc & $\%$ & Fem. & $\%$ & Total & $\%$ \\
\hline BR-174 & 167 & 64 & 78 & 71 & 245 & 67 & 317 & 76 & 81 & 72 & 398 & 75 \\
\hline Outras & 95 & 36 & 27 & 29 & 122 & 33 & 101 & 24 & 32 & 28 & 133 & 25 \\
\hline Total & 262 & 100 & 105 & 100 & 367 & 100 & 418 & 100 & 113 & 100 & 531 & 100 \\
\hline
\end{tabular}

19977

\begin{tabular}{|c|c|c|c|c|c|c|c|c|c|c|c|c|}
\hline \multicolumn{7}{|c|}{ Plasmodium vivax } & \multicolumn{6}{|c|}{ Plasmodium falciparum } \\
\hline Origem & Masc & $\%$ & Fem. & $\%$ & Total & $\%$ & Masc. & $\%$ & Fem. & $\%$ & Tota! & $\%$ \\
\hline BR-174 & 155 & 60 & 53 & 54 & 208 & 58 & 213 & 73 & 64 & 72 & 277 & 73 \\
\hline Outras & 103 & 40 & 46 & 46 & 149 & 42 & 80 & 27 & 25 & 28 & 105 & 27 \\
\hline Total & 258 & 100 & 99 & 100 & 357 & 100 & 293 & 100 & 89 & 100 & 382 & 100 \\
\hline
\end{tabular}

(*) - Os dados foram obtidos no laboratório de malária (SUCAM) do Hospital de Moléstias Tropicais de Monaus.

parum. O número de casos é maior no sexo masculino que no feminino. As infecções por vivax foram de 520 para 204 nos dois anos 1976 e 1977 enquanto que por falciparum foram de 711 para 202, respectivamente. A taxa mais alta no sexo masculino pode ser atribuída a uma maior exposição "ocupacional" ao vetor. A maioria dos colonizadores são do sexo masculino (Hayes \& Ferraroni, $\mathrm{s} / \mathrm{d}$ ). A grande diferença na proporção da ma. lária falciparum, num total de 531 casos e vivax 367 casos em 1976, foi reduzida em 1977 para aproximadamente um mesmo número, ou seja 382 falciparum para 357 vivax.

$\mathrm{O}$ decréscimo no número de casos de intecção por $P$. falciparum poderia ser atribuído a um aumento de anticorpo adquirido, nos colonizadores e a tolerância à malária. A estação seca foi mais prolongada em 1976 que em
1977. O pico para os casos de infecções pelo P. falciparum em 1974, 1975 e 1977, ocorreu em outubro e o pico para o mesmo plasmódio no ano de 1976, onde a estação seca foi dois meses após, ocorreu em dezembro (gráfico 1).

Os casos de malária começam a aumentar no final da estação chuvosa e atinge um pico entre os meses de outubro e dezembro dependendo de cada ano (gráfico 1).

Quando a estação chuvosa prolonga-se os casos de malária começam o declínio no final da estação, mas quando a estação chuvosa é curta os casos de malária iniciam o decréscimo já na estação seca. Os casos de malária falciparum caem rapidamente (gráfico 2) e os casos de malária vivax apresentam um ressur. gimento nos meses de março a junho provavelmente devido a recaídas. O Anopheles darlingi, principal vetor de malária no Amazo. 


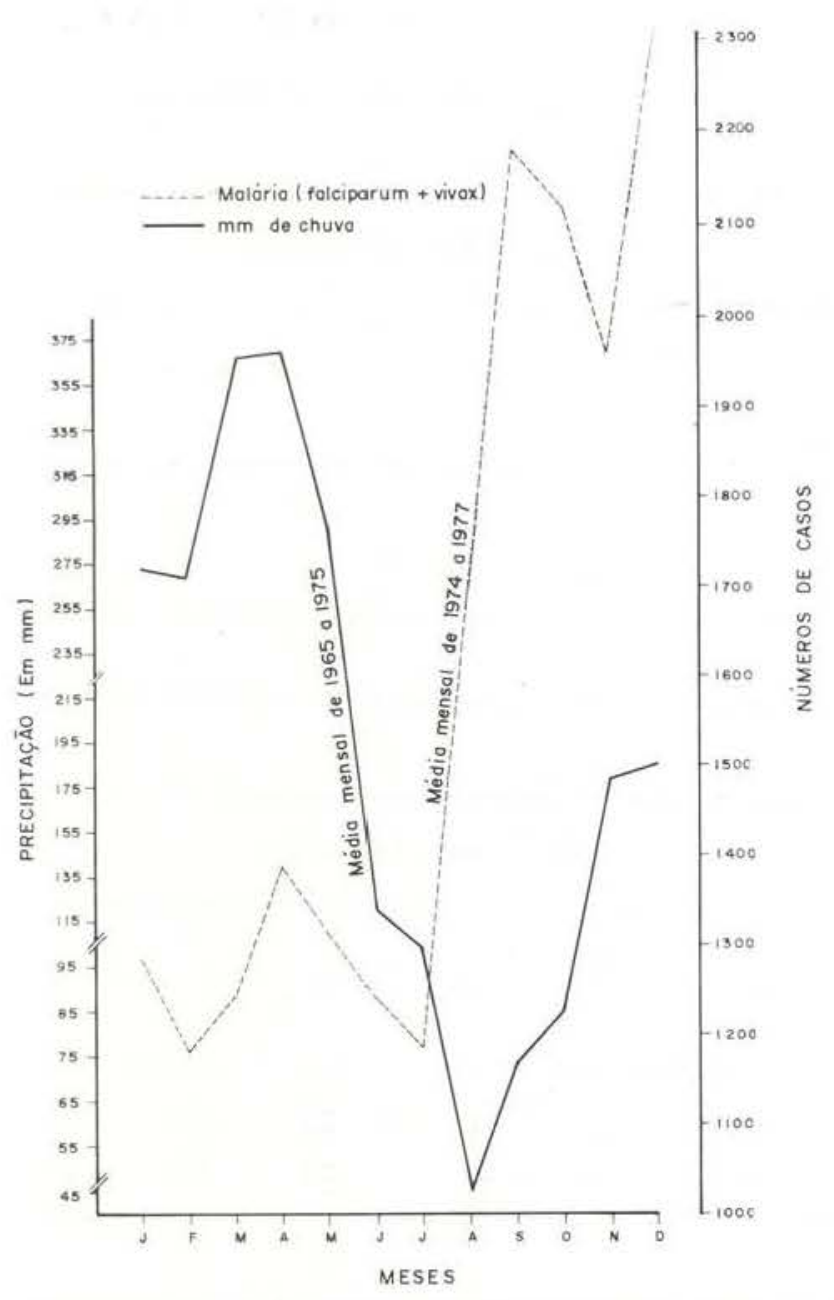

Gráfico 1 - Relação entre o periodo de chuva e a ocorrência da malária no Estado do Amazonas.

nas, apresenta um declínio com o prolongamento das chuvas (Hayes \& Charlwood, s/d).

Um grande número de fatores em ecologia humana pode influenciar nas taxas de malária de uma população, incluindo os fatores climáticos, assim como a ecologia do vetor. O comportamento do vetor picando o homem foi descrito muito detalhadamente por Hayes \& Charlwood (1978). Os diversos fatores relacionados à ecologia humana será apresentado no trabalho seguinte (Hayes \& Ferraroni, s/d) e a relação dos casos de malária com os níveis higrométricos, cheias dos grandes rios da bacia Amazônica, estação seca e chuvosa será apresentado num trabalho seguinte (Ferraroni \& Marzochi, s/d).

A população humana com malária (diagnosticada e tratada não hospitalizada) parece ser praticamente a mesma para ambos os se xos e ambas as espécies de plasmódios, equivalendo essas proporções para os anos de 1976 e 1977 (gráfico 2). É claro que existe um número maior de casos de infecção pelo P. falciparum no ano de 1976 do que em 1977. sendo também a maior taxa do sexo masculino. O volume de casos para ambas as espécies aumentam com a idade. Na malária vi. vax o maior número de casos aparece na faixa etária de 20 a 29 anos do sexo feminino, representando um pico bem delimitado, enquanto que no sexo masculino, também existe esse pico, mas com uma diferença bem distinta, onde o número de casos apresenta um p:co declinado gradualmente e não bruscamente como no sexo feminino. No sexo masculino nas idades de 30 a 39 e 40 a 49 anos, continua a mostrar um número significante de infecção por malária vivax. Nós atribuímos a isto a exposição ocupacional (Hayes \& Ferraroni, s/d). O fato anteriormente explicado para a infecção do $P$. vivax se repete com a do $P$. falciparum. Comparando os gráficos de números 3 e 4 verificamos um contraste entre homens com vivax e falciparum, o modelo é inteiramente similar. Um oposto entre malária vivax e falciparum em mulheres é verificado (ver sexo feminino no gráfico 3 ), novamente um contraste evidente. Por que na faixa etária de 10 a 14 anos as mulheres apresentam um grande declínio na infecção por vivax em 1976 e falciparum em 1977, não sabemos o motivo deste fato, sem precedentes na literatura. Nas pacientes hospitalizadas (gráfico 5) as mulheres na idade de 10 a 14 anos também mostram a ocorrência de uma queda em ambas as espécies de plasmódios no acumulativo de 1974 a 1977, não encontramos explicação para isso na literatura. Nos grupos etários seguintes ao IV, é claramente evidenciado que as mulheres constituem a menor proporção de hospitalização, o que consiste numa menor porcentagem de infecção (gráficos 3 e 4) e menor exposição ocupacional, isto também é evidente no gráfico 5 . A infecção por $P$. falciparum é responsável por três vezes a mais que aquela por $P$. vivax no total de pacientes hospitalizados, o que pods ser visto também na tabela 2, mostrando ainda que as hospitalizações (dias de interna- 


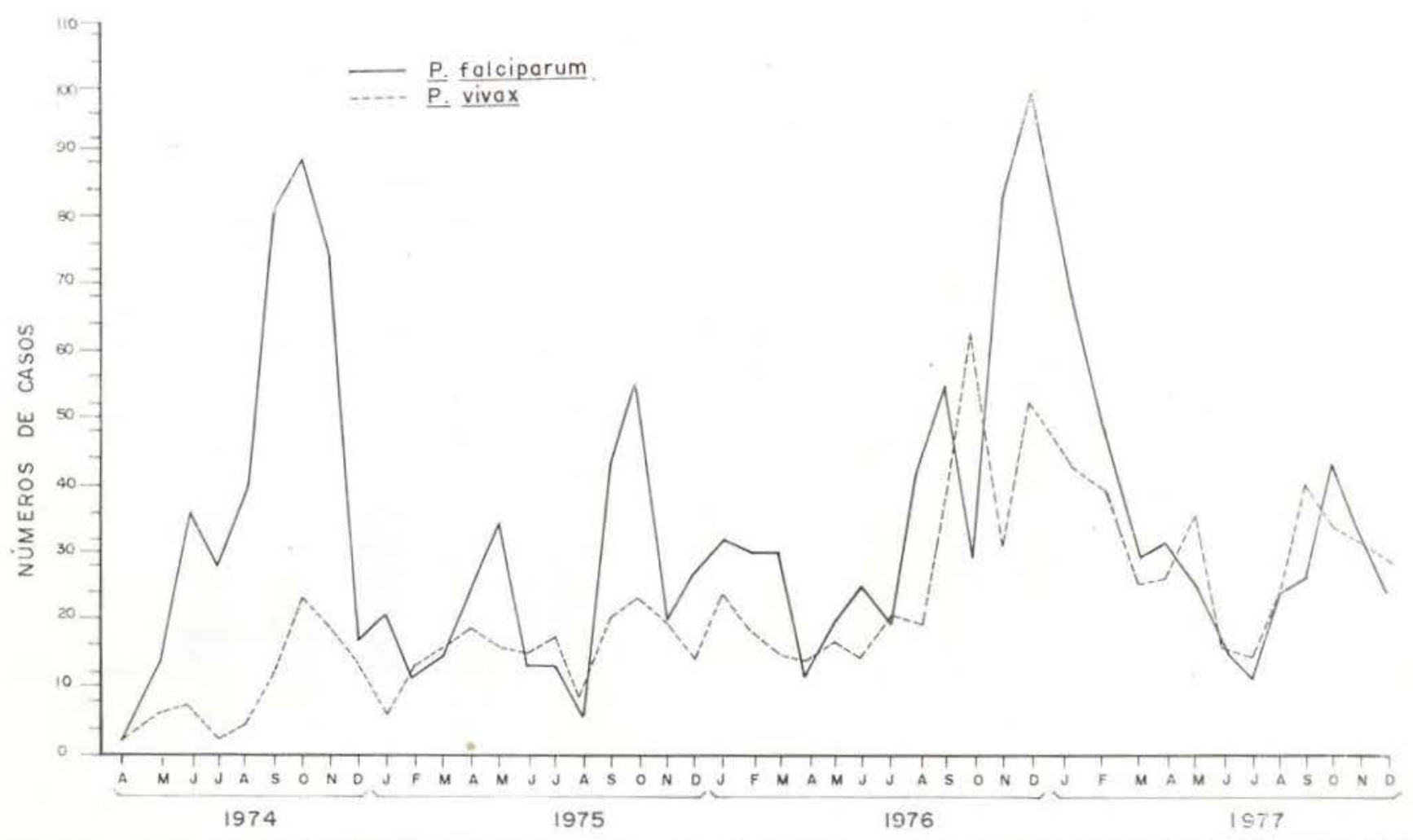

Gráfico 2 - Casos de malária humana diagnosticado no Hospital Tropical de Manaus no período de abril de 1974 a dezembro de 1977 .

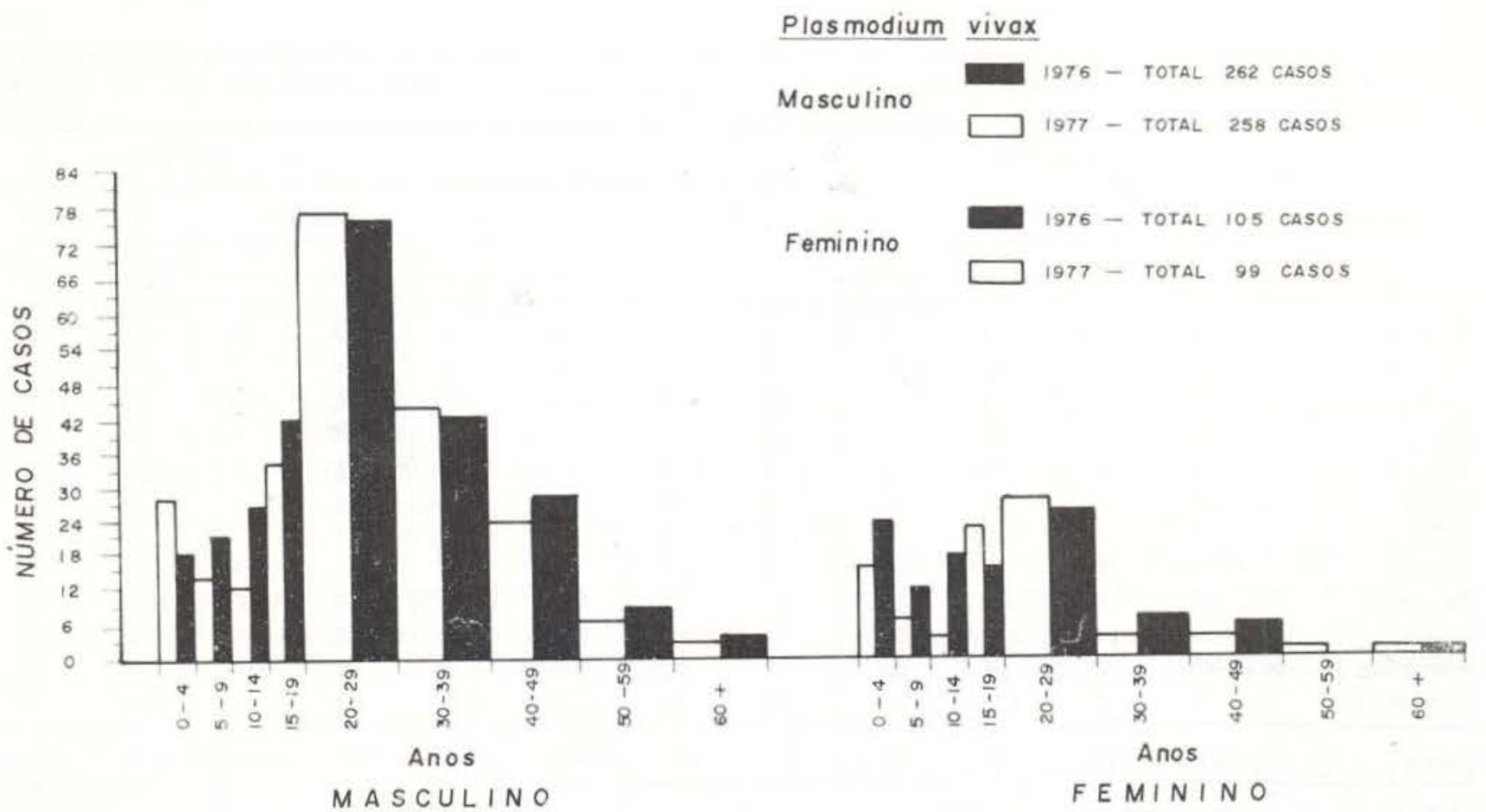

Gráfico 3 - Distribuição por idade e sexo dos casos de malária humana (Plasmodium vivax) diagnosticado no Hos. pital de Moléstias Tropicais de Manaus, nos anos de 1976 a 1977. 


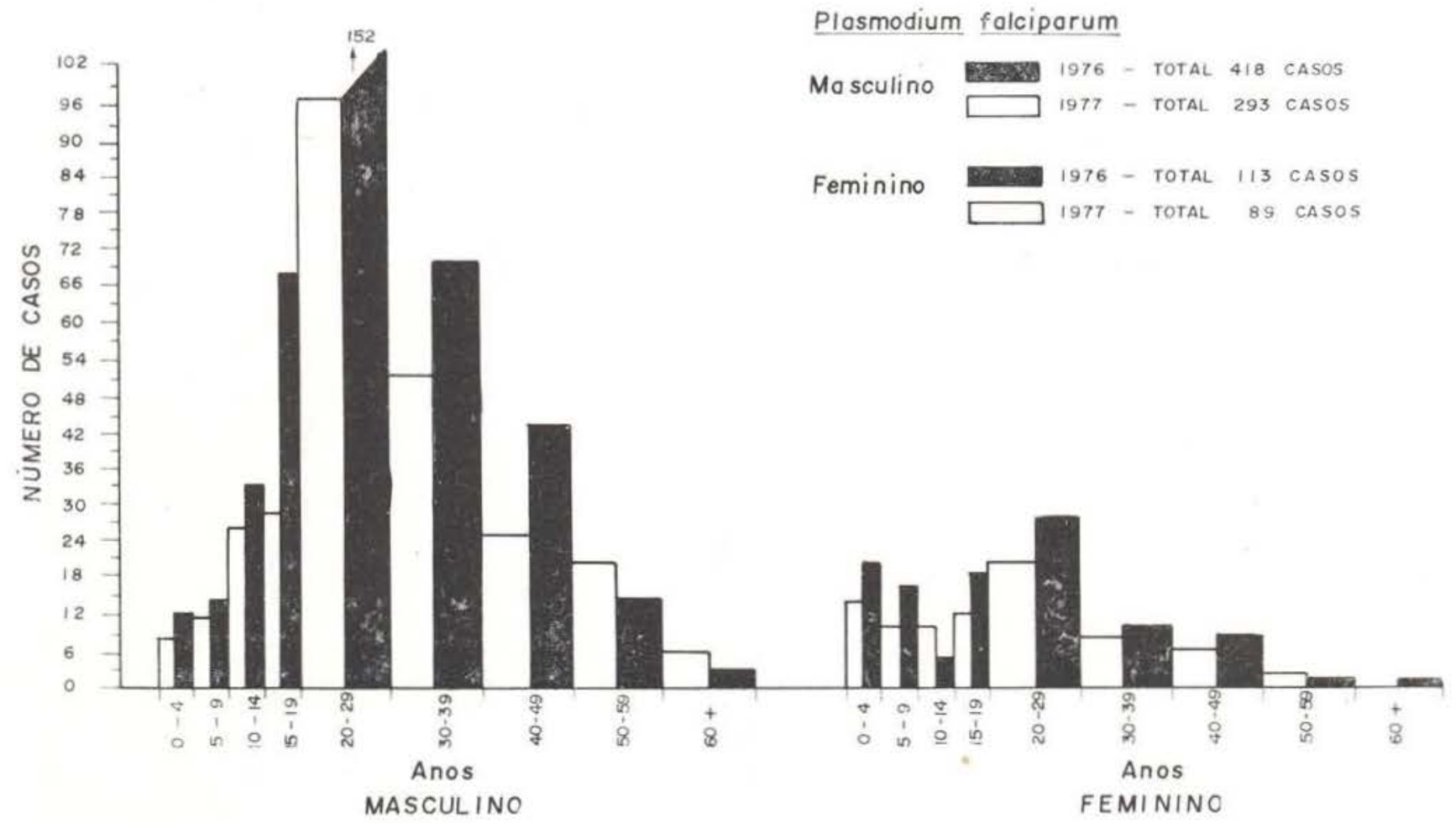

Gráfico 4 - Distribuiçăo por idade e sexo dos casos de malária humana (Plasmodium falciparum) diagnosticado no Hospital de Moléstias Tropicais de Mianaus, nos anos de 1976 a 1977.

TABELA 2 - Números de dias de hospitalização de pacientes com malária internados no Hospital de Moléstias Tropicais de Manaus relacionando o mês e espécie de plasmódios nos anos 1975-1976 e 1977. SAME* do H.M.T.

\begin{tabular}{|c|c|c|c|c|c|c|c|c|c|c|c|c|c|}
\hline \multirow{3}{*}{\multicolumn{2}{|c|}{$M$ ê $s$}} & \multicolumn{4}{|c|}{1975} & \multicolumn{4}{|c|}{1976} & \multicolumn{4}{|c|}{1977} \\
\hline & & \multicolumn{2}{|c|}{ vivax } & \multicolumn{2}{|c|}{ falciparum } & \multicolumn{2}{|c|}{ vivax } & \multicolumn{2}{|c|}{ falciparum } & \multicolumn{2}{|c|}{ vivax } & \multicolumn{2}{|c|}{ falciparum } \\
\hline & & Masc. & Fem. & Fem. & Masc. & Fem. & Masc. & Fem. & Masc. & Masc. & Fem. & Masc. & Fem. \\
\hline Janeiro & & 4 & 0 & 41 & 14 & 7 & 2 & 85 & 24 & 64 & 29 & 260 & 153 \\
\hline Fevereiro & & 10 & 0 & 41 & 0 & 4 & 11 & 87 & 42 & 26 & 7 & 226 & 222 \\
\hline Março & & 59 & 32 & 25 & 0 & 15 & 8 & 74 & 36 & 10 & 4 & 132 & 55 \\
\hline Abril & & 31 & 0 & 52 & 21 & 16 & 0 & 49 & 28 & 56 & 0 & 138 & 32 \\
\hline Maio & & 11 & 0 & 46 & 8 & 0 & 3 & 99 & 38 & 16 & 6 & 227 & 72 \\
\hline Junho & & 27 & 0 & 25 & 41 & 0 & 6 & 82 & 12 & 8 & 6 & 82 & 3 \\
\hline Julho & & 9 & 0 & 25 & 0 & 26 & 9 & 105 & 28 & 1 & 23 & 74 & 40 \\
\hline Agosto & & 23 & 16 & 47 & 0 & 9 & 0 & 151 & 76 & 21 & 10 & 69 & 31 \\
\hline Setembro & & 17 & 0 & 69 & 22 & 12 & 0 & 121 & 72 & 17 & 49 & 116 & 62 \\
\hline Outubro & & 49 & 13 & 187 & 56 & 77 & 11 & 221 & 49 & 15 & 21 & 133 & 81 \\
\hline Novembre & & 33 & 2 & 61 & 42 & 76 & 31 & 390 & 102 & 5 & 24 & 244 & 57 \\
\hline Dezembro & & 3 & $\epsilon$ & 129 & 25 & 19 & 62 & 305 & $4 C$ & 13 & 11 & 272 & 44 \\
\hline Total & & 276 & EQ & 748 & 229 & 261 & 143 & 1769 & 547 & 252 & 190 & 1972 & 852 \\
\hline . & $=$ & \multicolumn{2}{|c|}{$\begin{array}{c}\text { Total } \\
\text { vivax } \\
345\end{array}$} & \multicolumn{2}{|c|}{$\begin{array}{c}\text { Total } \\
\text { falciparum } \\
977\end{array}$} & \multicolumn{2}{|c|}{$\begin{array}{c}\text { Total } \\
\text { vivax } \\
404\end{array}$} & \multicolumn{2}{|c|}{$\begin{array}{c}\text { Tota! } \\
\text { falciparum } \\
2316\end{array}$} & \multicolumn{2}{|c|}{$\begin{array}{c}\text { Total } \\
\text { vivax } \\
442\end{array}$} & \multicolumn{2}{|c|}{$\begin{array}{c}\text { Total } \\
\text { falciparum } \\
2824\end{array}$} \\
\hline
\end{tabular}

(*) - Serviço de Arquivo Médico e Estatístico. 


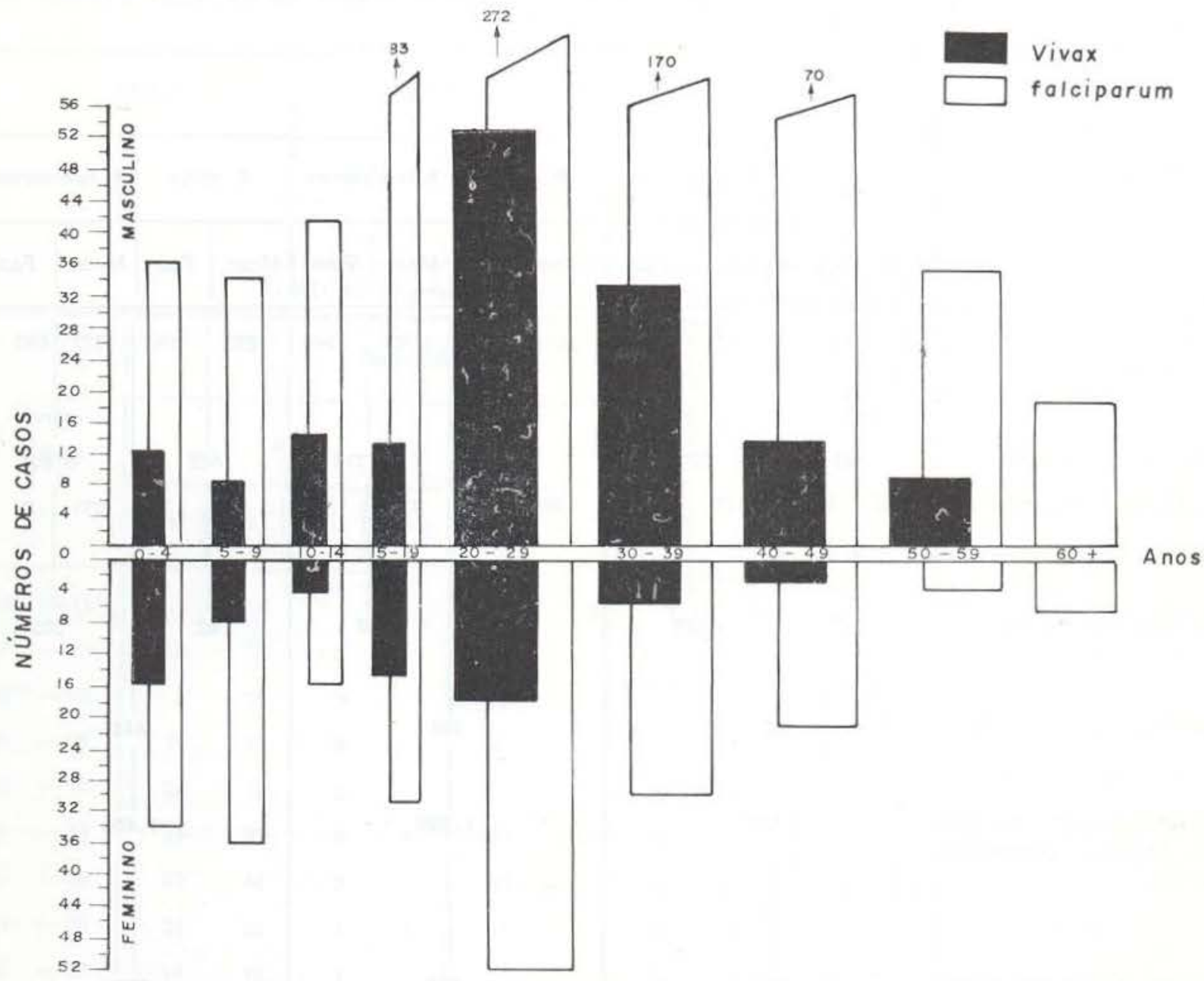

Grafico 5 - Comparação dos casos de malária falciparum e vivax, por idade e sexo nos pacientes hospitalizados no Hospital de Moléstias Tropicais de Manaus, no período de abril/74 a dezembro de 1977.

mentos) tem aumentado do ano de 1975 para 1977. Isto deve-se em parte a maior disponibilidade de leitos para malária, graças aos esforços que a Direção do Hospital de Moléstias Tropicais de Manaus tem realizado com o intuito de combater a referida endemia em nosso Estado.

A porcentagem de leitos, ocupados por pacientes, sofreu mudanças pouco significativas de 1976 para 1977 (29\% e $30 \%$ respectivamente). A porcentagem quase dobrou de 1975 $(17 \%)$ para $1976(29 \%)$, (tabela 3) com maior prevalência para os casos de malária falciparum, isto deve-se ao fato do $P$. falciparum apresentar maior poder patogênico pois invade hemácias em todas as idades, alcançando às vezes mais de $50 \%$ de parasitemia $(50 \%$ do total das hemácias), enquanto que o $P$. vivax invadindo preferencialmente hemácias jovens, dificilmente atinge $10 \%$ de parasitemia.

É interessante notar que a mortalidade da malária atinge mais a faixa etária jovem, do total de óbitos ocorridos no Hospital de Moléstias Tropicais de Manaus nos anos de 1974 a 1977 verificamos que mais de $38 \%$ ocorreu nos pacientes com idades inferiores a 15 anos (tabela 5).

O outro hospital de Manaus que trata casos de malária rotineiramente é o Hospital Geral de Manaus (Hospital Militar), onde verificamos que dentre todas as doenças tropi- 
TABELA 3 - Número de pacientes/dias para a infecção malárica, internados no Hospital de Moléstias Tropicais de Manaus, Amazonas - Brasil.

\begin{tabular}{|c|c|c|c|c|c|c|c|c|c|c|c|c|}
\hline \multirow{3}{*}{ Especificação } & \multicolumn{4}{|c|}{1975} & \multicolumn{4}{|c|}{1976} & \multicolumn{4}{|c|}{1977} \\
\hline & \multicolumn{2}{|c|}{ P. vivax } & \multicolumn{2}{|c|}{ P. falciparum } & \multicolumn{2}{|c|}{ P. vivax } & \multicolumn{2}{|c|}{ P. falciparum } & \multicolumn{2}{|c|}{ P. vivax } & \multicolumn{2}{|c|}{ P. falciparum } \\
\hline & Masc & Fem. & Masc & Fem. & Masc & Fem & Masc & Fem. & Masc. & Fem. & Masc & Fem. \\
\hline $\begin{array}{l}\text { Número de dias rospitalizados } \\
\text { por sexo e espécie }\end{array}$ & $2 \pi 6$ & 69 & 748 & 229 & 261 & 143 & 1769 & 547 & 252 & 193 & 1972 & 852 \\
\hline Total de dias por espécie & 3 & 45 & & & 40 & 04 & & 316 & 44 & & & .824 \\
\hline $\begin{array}{l}\text { Número de pacientes com ma- } \\
\text { lária }\end{array}$ & 36 & 10 & 110 & 33 & 38 & 14 & 237 & 63 & 49 & 33 & 253 & 99 \\
\hline Número total por espécie & 4 & 6 & & & 52 & & 30 & & 82 & & & 362 \\
\hline $\begin{array}{l}\text { Número total das duas espé } \\
\text { cies }\end{array}$ & & & 89 & & & & 58 & & & & 44 & \\
\hline $\begin{array}{l}\text { Número total de pacientes com } \\
\text { doenças tropicais hospitaliza- } \\
\text { dos por ano }\end{array}$ & & & 142 & & & & 250 & & & & 484 & \\
\hline $\begin{array}{l}\text { Porcentagem de tempo consu- } \\
\text { mido em todos os casos de } \\
\text { malária hospitalizados. }\end{array}$ & & 17 & $\%$ & & & & $29 \%$ & & & & $0 \%$ & \\
\hline
\end{tabular}

TABELA 4 - Pacientes internados no Hospital Geral de Manaus acometidos de doenças infecciosas no ano de 1977, relacionando os civis e militares. SAME ${ }^{\circ}$ do HGeM.

\begin{tabular}{|c|c|c|c|c|}
\hline Diagnóstico & Militar & Civil & Total & $\%$ \\
\hline Doenças venereas & 99 & 3 & 112 & 30,0 \\
\hline Leishmaniose & 59 & 1 & 60 & 17,3 \\
\hline Intoxicação alimentar & 31 & - & 31 & 9,0 \\
\hline Varicela & 25 & 1 & 26 & 7,5 \\
\hline Malária & 16 & 5 & 21 & 6,0 \\
\hline Hansenicse & 8 & 2 & 10 & 2,9 \\
\hline Tuberculose & 6 & 4 & 10 & 2.9 \\
\hline Outras & 41 & 43 & 84 & 24,4 \\
\hline TOTAL & 285 & 59 & 344 & 100,0 \\
\hline
\end{tabular}

(*) - Serviço de Arquivo Médico e Estatística. cais a malária constitui $6 \%$ dos casos hospitalizados. A média de avariaçăo do tempo de permanência em dias no Hospital Militar para os pacientes com malária foi de 12,44 para as infecções por $P$. falciparum e 8,59 para o $P$. vivax. O curto período de tempo hospitalizado para o pessoal militar pode ser atribuído ao padrão de vida dos mesmos. Os pacientes internados no Hospital de Moléstias Tropicais de Manaus, em sua grande maioria tem procedência da BR-174, são geralmente caboclos de baixo padrão sócio-econômico nos quais a própria situação dietética poderia ser conside. rada inferior à dos militares e também a faixa etária dos pacientes militares é bem delimita. da estando compreendida na sua quase totalidade entre 18 a 24 anos de idade. 
TABELA 5 - Óbitos ocorridos pela infecção malárica no período de abril de 1974 a dezembro de 1977 no Hospital de Moléstias Tropicais de Manaus, SAME

\begin{tabular}{|c|c|c|c|c|}
\hline M ês & Idade & Sexo & $\begin{array}{l}\text { Espécie de } \\
\text { Plesmódio }\end{array}$ & Ano \\
\hline Maio & 19 & Masc. & falciparumi & 1974 \\
\hline Setembro & 8 & Fem. & $n$ & $"$ \\
\hline Setembro & 3 & Fem. & $n$ & $"$ \\
\hline Outubro & 35 & Fem. & $"$ & $"$ \\
\hline Novembro & 24 & Masc. & " & $"$ \\
\hline Novembro & 25 & Masc. & $"$ & " \\
\hline Dezembro & 7 & Masc. & $"$ & $n$ \\
\hline Fevereiro & 23 & Masc. & falciparum & 1975 \\
\hline Abril & 47. & Masc. & $"$ & $"$ \\
\hline Setembro & $6 * *$ & Fem. & $"$ & $"$ \\
\hline Outubro & 39 & Masc. & $"$ & $n$ \\
\hline Outubro & 70 & Masc. & $n$ & " \\
\hline Novembro & 3 & Fem. & $n$ & $"$ \\
\hline Dezembro & 27 & Masc. & $"$ & $"$ \\
\hline Julho & 16 & Masc. & falciparum & 1976 \\
\hline Agosto & 12 & Masc. & $"$ & $"$ \\
\hline Setembro & 28 & Fem. & $"$ & $"$ \\
\hline Outubro & 57 & Masc. & $"$ & $"$ \\
\hline Novembro & 15 & Fem. & $"$ & $"$ \\
\hline Dezembro & 43 & Masc. & $"$ & $"$ \\
\hline Dezembro & 27 & Masc. & $"$ & $"$ \\
\hline Fevereiro & 10 & Fem. & falciparum & 1977 \\
\hline Fevereiro & 38 & Masc. & $"$ & $"$ \\
\hline Outubro & 14 & Fem. & $"$ & $n$ \\
\hline Novembro & 2 & Fem. & $"$ & $"$ \\
\hline Dezembro & 35 & Masc. & $"$ & $n$ \\
\hline
\end{tabular}

(*) - Serviço de Arquivo Médico e Estatistico.

(**) - A idade está representada em meses.

\section{AGRADECIMENTOS}

Os autores agradecem ao Dr. Heitor Vieira Dourado, diretor do Hospital de Moléstias Tropicais de Manaus, e Dr. Antonio Maia Barbosa, diretor do Hospital Geral de Manaus, por colocarem os dados do SAME à nossa disposição .

\section{SUMMARY}

Overall rates of malaria for Amazonas have be dropping since 1973. An exception to this trend are the newly opened roads, BR-174 (Manaus/Boa Vista) and BR-319 (Manaus/Porto Velho). BR-174 has been an endemic focus of drug resistant falciparum malaria and the vector Anopheles darlingi exhibit behavior resistance to DDT. Since 1974 when the Hospital de Moléstias Tropicais de Manaus first opened, nearly all hospitalized cases of malaria have been treated at this facility, Malaria cases (out patients) as well as hospitalized cases were studied to determine the nature of malaria. Data is presented by species of plasmodium, month, year and by age and sex. Data is also presented from the military Hospital. Individuals from BR-174 constitued over $65 \%$ of all malaria cases seen by the hospital. This study is important because of its implications for future development of the Amazon.

\section{BIBLIOGRAFIA}

Anuário Estatí́stico do Governo do Estado dO AMAZONAS

1975 - Secretaria de Planejamento e coordenação geral, Comissão de Desenvolvimento do Estado do Amazonas, Manaus - 1976.

FERraroni, J.J. \& Marzochi, M.A.C.

s/d - Periodicidade estacional da malária humana em Manaus, Amazonas (em preparaçăo) .

FERRARONI, J.J.; WAKI, S. \& SUZUKI, M.

1977 - Resistência do Plasmodium falciparum as cloroquinas no Estado do Amazonas, detectada pelo método IN VITRO. Acta Ama. zonica, $7(1): 147-148$.

Hayes, J. \& Charlwood, D.

1977 - O Anopheles darlingi evita o DDT numa área de malária resistente a drogges. Acta Amazonica, $7(2): 289$.

$\mathrm{s} / \mathrm{d}$ - Seasonal changes in Anopheles darlingi populations in an endemic area of malaria in the Amazon (em preparação).

HAYES, J. \& FERRARONI, J.J.

$\mathrm{s} / \mathrm{d}$ - Epidemic malaria on a newly opened road in the Amazon (em preparação).

MINISTÉRIO DA SAÚDE

1973 - Ministério da Saúde na Amazônia - Saúde e Saneamento na Transamazônica.

Organização Mundial da Saúde (OMS)

1975 - Manual do diagnóstico microscópico da malária. Publicação Científica (276).

(Aceito para publicação em 4/05/78) 
TABELA 6 - Parasitemia dos casos de malária humana em Manaus relacionando a espécie de Plasmódio e grupo etário no período de janeiro a dezembro de 1976 e 1977. SAME1 do Hospital de Moléstias Tropicais de Manaus.

\begin{tabular}{|c|c|c|c|c|c|c|c|c|c|c|c|c|c|c|c|c|c|c|c|c|c|c|c|c|c|c|c|c|}
\hline \multirow{3}{*}{$\begin{array}{l}\text { Grupo } \\
\text { Etário }\end{array}$} & \multicolumn{8}{|c|}{ Plasmodium vivax } & \multicolumn{8}{|c|}{ Plasmodium falciparum } & \multirow{2}{*}{\multicolumn{4}{|c|}{$\frac{\begin{array}{c}\text { TO TAL } \\
\text { P. vivax + falciparum }\end{array}}{1976}$}} & \multirow{3}{*}{$\begin{array}{l}T \\
0 \\
T \\
4 \\
i\end{array}$} & \multirow{3}{*}{$\%$} & \multirow{2}{*}{\multicolumn{4}{|c|}{$\begin{array}{c}\begin{array}{c}\text { TOTAL } \\
\text { P. vivax }+ \text { falciparum }\end{array} \\
1977\end{array}$}} & \multirow{3}{*}{$\begin{array}{l}T \\
0 \\
T \\
A \\
L\end{array}$} & \multirow{3}{*}{$\%$} \\
\hline & \multicolumn{4}{|c|}{1976} & \multicolumn{4}{|c|}{1977} & \multicolumn{4}{|c|}{1976} & \multicolumn{4}{|c|}{1977} & & & & & & & & & & & & \\
\hline & \pm & \pm \pm & \pm \pm \pm & \pm \pm \pm \pm & \pm & \pm \pm & \pm \pm \pm & \pm \pm \pm \pm & \pm & \pm \pm & \pm \pm \pm & $\pm \pm \pm \pm \cdot$ & \pm & \pm \pm & \pm \pm \pm & \pm \pm \pm \pm & \pm & \pm \pm & \pm \pm \pm & \pm \pm \pm \pm & & & \pm & \pm \pm & \pm \pm \pm & \pm \pm \pm \pm & & \\
\hline $00-11^{*}$ & 1 & 2 & 1 & & 1 & 9 & 2 & 1 & 1 & 2 & 1 & & 1 & 2 & 1 & & 2 & 4 & & & 8 & 0,9 & 2 & 11 & 3 & 1 & 17 & 2,2 \\
\hline $12 \quad-23$ & 5 & 3 & 1 & & 3 & 3 & 1 & & 2 & 1 & 2 & 1 & 1 & 1 & 1 & & 7 & 4 & 3 & 1 & 15 & 1,7 & 4 & 4 & 2 & & 10 & 1.3 \\
\hline $02^{* *}-04$ & 5 & 18 & 1 & & 7 & 14 & 4 & & 10 & 9 & 4 & & 6 & 8 & 1 & & 15 & 17 & . 5 & & 47 & 5,3 & 13 & 22 & 5 & & 40 & 5,1 \\
\hline $05-09$ & 11 & 19 & 4 & & 12 & 8 & 2 & & 20 & 8 & 5 & & 10 & 6 & 5 & 1 & 31 & 27 & 9 & & 67 & 7,4 & 22 & 14 & 7 & 1 & 44 & 5,7 \\
\hline $10-14$ & 22 & 19 & 2 & & 6 & 10 & 1 & & 11 & 8 & 8 & 1 & 19 & 15 & 2 & 1 & 33 & 27 & 10 & 1 & 71 & 7,9 & 25 & 25 & 3 & 1 & 54 & 6,9 \\
\hline $15-19$ & 21 & 31 & 2 & 1 & 21 & 27 & 4 & & 29 & 28 & 12 & 1 & 13 & 16 & 11 & & 50 & 59 & 14 & 2 & 125 & 13,9 & 34 & 43 & 15 & & 92 & 11,7 \\
\hline $20-29$ & 53 & 44 & 3 & & 30 & 61 & 3 & & 67 & 76 & 37 & 3 & 48 & 51 & 17 & 1 & 120 & 120 & 40 & 3 & 283 & 31,5 & 78 & 112 & 20 & 1 & 211 & 26,9 \\
\hline $30-39$ & 31 & 20 & 1 & 1 & 21 & 21 & 2 & & 34 & 29 & 14 & 1 & 25 & 30 & 5 & 1 & 15 & 49 & 15 & 2 & 131 & 14,6 & 46 & 51 & 7 & 1 & 105 & 13,4 \\
\hline $40-49$ & 14 & 12 & 1 & & 15 & 12 & & & 24 & 23 & 7 & 2 & 12 & 15 & 3 & 1 & 39 & 35 & 8 & 2 & 83 & 9,2 & 27 & 27 & 3 & 1 & 58 & 7,5 \\
\hline $50-59$ & 3 & 2 & 1 & & 2 & 3 & & & 9 & 8 & 1 & & 9 & 11 & 2 & & 12 & 10 & 2 & & 24 & 2,7 & 11 & 14 & 2 & & 27 & 3,4 \\
\hline $50+$ & 2 & 1 & & & 3 & 2 & & & 4 & & 1 & & 3 & 3 & & & 6 & 1 & 1 & & 8 & 09 & 6 & 5 & & & 11 & 1,4 \\
\hline $\begin{array}{l}\text { Idade } \\
\text { ignorada }\end{array}$ & 1 & 5 & 3 & & 26 & 40 & & & 11 & 9 & 5 & 2 & 23 & 22 & 2 & 1 & 12 & 14 & 8 & 2 & 26 & 4,0 & 49 & 62 & 2 & 1 & 114 & 14,5 \\
\hline Total & 169 & 176 & 20 & 2 & 147 & 210 & 19 & 1 & 222 & 201 & 97 & 11 & 170 & 180 & 50 & 6 & 391 & 377 & 117 & 13 & 898 & 100,0 & 317 & 390 & 69 & 7 & 783 & 100,0 \\
\hline
\end{tabular}

$(*)$ - Meses

1 ) - Serviço de Arquivo Médico e Estatístico.

$( \pm)-01$ parasita por campo em gota espessa, segundo o critério da O.M.S

( ++ ) - 02 a 20 porasitas por campo em gota espessa, segundo o critério do O.M.S

( ++++$)-21$ a 200 parasitas por campo em gota espessa, segundo o critério da O.M.S.

Em 1976 - 7 casos infecçöes mistas; Em 1977 - 8 casos de infecções mistas Estes dados não foram includos na tabela

Aspectos. . 